\title{
Recurrent Bioprosthetic Mitral Valve Thrombosis Treated with Anticoagulation
}

\author{
Robin Fernandes ${ }^{1}$, Anubodh Varshney ${ }^{2}$, and Jayashri Aragam ${ }^{1}$ \\ ${ }^{1}$ Boston VA Medical Center \\ ${ }^{2}$ Brigham and Women's Hospital Division of Cardiology
}

July 20, 2020

\begin{abstract}
Bioprosthetic valve thrombosis (BPVT) is more common than previously thought and likely underreported. BPVT can be accurately diagnosed with cardiac imaging and treated successfully with anticoagulation, thus preventing re-operation. We hereby report a case of recurrent BPVT in the mitral position successfully treated with anticoagulation along with review of literature.
\end{abstract}

\section{Introduction:}

The mechanism of Bioprosthetic valve (BPV) dysfunction is predominantly due to structural deterioration. Bioprosthetic valve thrombosis (BPVT) has traditionally been considered a rare event. However, recent data suggest that BPVT is not uncommon and in fact an increasingly recognized entity. The true incidence of BPVT may be underestimated as most cases remain under diagnosed. The diagnosis can be challenging due to low general awareness of the condition. A high degree of clinical suspicion and formal echocardiographic criteria can accurately diagnose BPVT. Early diagnosis remains crucial as it can be successfully treated with anticoagulation.

\section{Case Report:}

A 72-year-old male presented to the emergency room with one-week history of progressive exertional dyspnea and lower extremity edema. His past medical history included mitral valve replacement (MVR) eight years ago [27mm Carpentier-Edward (CE) Magna] for severe mitral regurgitation (MR) due to myxomatous mitral valve disease, paroxysmal atrial fibrillation (pAF), and non-ischemic cardiomyopathy with LVEF 30-35\% s/p dual chamber implantable cardioverter-defibrillator (ICD). Two years prior to the current admission, he presented with multiple ICD shocks secondary to atrial tachycardia. Work up revealed multiple echodensities on the mitral bioprosthetic valve with BPV stenosis and a transmitral mean gradient of $7 \mathrm{~mm} \mathrm{Hg}$ at heart rate 61 beats per min (bpm). After a detailed workup he was diagnosed with non-bacterial thrombotic endocarditis [Figure 1, Video 1-2]. He was treated with unfractionated heparin (UFH) for two weeks. However, due to lack of improvement, he underwent re-do MVR with a similar valve (27mm CE Magna) and was discharged on warfarin with international normalized ratio (INR) goal 2-3. One month prior to the current presentation, warfarin was interrupted perioperatively for total right hip replacement surgery.

At the time of his current admission, physical examination revealed a chronically ill appearing man with blood pressure 121/94 mmHg, irregularly irregular rhythm with heart rate of $84 \mathrm{bpm}$, normal S1 and S2 without murmurs, jugular venous pressure $12 \mathrm{~cm}$ of water, bibasilar crackles, and bilateral $4+$ pitting pedal edema. He was started on intravenous (IV) diuretics and admitted to the cardiology inpatient service. Laboratory evaluation included normal blood counts and metabolic panel, elevated B-type natriuretic peptide (2,751 $\mathrm{pg} / \mathrm{ml}$ ) and INR (2.3). Transthoracic echocardiogram (TTE) showed LVEF 30-35\%, mitral BPV leaflet 
thickening with mean gradient of $10 \mathrm{mmHg}$ at heart rate of $65 \mathrm{bpm}$, and multiple echo densities suspicious for vegetation or thrombus [Figure 2A \& 2B, Video 3]. Transesophageal echocardiogram (TEE) revealed severe BPV leaflet thickening with restricted motion, a large echo density encompassing both leaflets with a mobile component measuring $1.4 \times 0.4 \mathrm{~cm}$ and a mean gradient of $9.2 \mathrm{mmHg}$ at heart rate of $67 \mathrm{bpm}$ [Figure 2C, 2D, 3A, 3B, Video 4-6)]. A detailed laboratory work-up including infectious, rheumatologic, immunologic, and allergic (for bovine pericardial valve) tests was unremarkable. Given echocardiographic findings of BPV stenosis and echodensities and an extensive negative work up for other etiologies, he was treated empirically for BPVT with UFH and eventually transitioned to warfarin with an increased INR goal of 2.5-3.5. After three months of uninterrupted anticoagulation, a repeat TEE showed complete resolution of $\mathrm{BPV}$ thickening and echo density with significant reduction in the mean transvalvular gradient to $4 \mathrm{mmHg}$ at heart rate of $65 \mathrm{bpm}$ [Figure 4, and Video 7-8], indicating that the cause of the patient's initial presentation was likely BPVT. The patient is currently asymptomatic and is followed clinically and with regular surveillance TTE.

\section{Discussion:}

The true incidence of BPVT remains uncertain; estimates range from less than $0.5 \%$ to over $6 \%$ of BPV recipients depending on the mode of diagnosis (pathology vs imaging) and length of follow up ${ }^{1}$. BPV dysfunction due to thrombosis is commonly mistaken with 'valve degeneration', leading to underreporting. Though rare, BPVT is a clinically important entity and can occur in all four valve locations. BPVT is distinguished from BPV degeneration based on various echocardiographic criteria. BPVT presents with increased cusp thickness, reduced cusp mobility, and less severe regurgitation, whereas BPV degeneration is associated with calcified cusps, reduced mobility, and significant regurgitation.

The mechanisms underlying BPVT are incompletely understood, but involve blood flow perturbation resulting in high wall shear stress and increased blood stasis, plasma protein adsorption leading to activation of hemostatic factors, and patient related factors including hypercoagulable states, anemia, renal insufficiency, obesity, diabetes mellitus, smoking, low cardiac output and periprocedural trauma. Suboptimal anticoagulation in patients taking oral anticoagulation $(\mathrm{OAC})$ and $\mathrm{pAF}$ are additional risk factors for BPVT ${ }^{1}$.

BPVT presentation may vary from incidental imaging findings without symptoms to syncope, dyspnea, heart failure, or cardiogenic shock from valve obstruction. Diagnosis is typically made by echocardiography. A model consisting of three echocardiographic predictors increased the sensitivity and specificity of diagnosing BPVT in the setting of concordant clinical features: 1) $50 \%$ increase in transvalvular gradients compared with baseline within five years of surgery, in the absence of a high cardiac output state; 2 ) increased cusp thickness (>2 mm), especially on the downstream aspect of the valve; and 3) abnormal cusp mobility ${ }^{2}$. Although TTE is helpful, performing TEE is strongly recommended when clinical suspicion is high to facilitate expeditious diagnosis.

Although current 2017 ACC/AHA and ESC guidelines recommend oral anticoagulation for only the first three months following surgical BPV replacement in the absence of risk factors ${ }^{34}$, our case highlights the fact that risk of BPVT is not limited to first three months after implantation. Several studies have reported that BPVT may occur late after initial implantation and should be suspected in the appropriate clinical scenario. Among 149 patients who underwent mitral BPV implantation at a single center, a retrospective review of TEE's identified 9 patients $(6 \%)$ with BPVT and median time from implantation to diagnosis was 12 months ${ }^{5}$. Another study identified 46 cases (11.6\%) of histologically proven BPVT among 397 patients who underwent BPV explantation. The median time to explantation was 24 months, with $15 \%$ of cases occurring more than 5 years after valve placement ${ }^{2}$.

Anticoagulation is the mainstay treatment for BPVT in hemodynamically stable patients. Early diagnosis of BPVT is crucial as most patients respond to anticoagulation and BPVT resolves completely, thus avoiding need for repeat valvular intervention. Several studies have reported successful resolution of BPVT when treated with VKA (vitamin K antagonist) ${ }^{5678}$. A prospective evaluation of warfarin showed that anticoagulation was effective in $83 \%$ of patients with suspected BPVT, and most responded within 3 months ${ }^{7}$. A 
recent study by Petrescu et al. reported long term outcomes of anticoagulation in 83 patients treated with warfarin for suspected BPVT. Echocardiographic parameters normalized in $75 \%$ of patients within three months. However, warfarin-treated patients had significant higher rates of major bleeding compared with matched controls. Additionally, BPVT recurred in $23 \%$ of warfarin responders after a median of 23 months, and all but one patient with recurrent BPVT responded to anticoagulation. Thus, longer term or even indefinite anticoagulation with warfarin could be considered after an initial BPVT episode while balancing bleeding risks ${ }^{8}$. In retrospect, we believe that our patient's presentation of BPV stenosis two years prior to the current presentation was likely BPVT. At that time, an extended trial of anticoagulation may have obviated the need for redo MVR.

While current 2017 ACC/AHA guidelines do not recommend routine surveillance with TTE until after 10 years of bioprosthetic valve implantation in the absence of symptoms, our case highlights that early diagnosis and management of BPVT is critical. Thus, consideration should be given to regular TTE monitoring for the development of BPVT in high risk cohorts. Determining the optimal frequency of TTE monitoring for $\mathrm{BPV}$ recipients requires further investigation.

\section{Conclusions:}

BPVT may occur late after valve implantation and should not be confused for valve degeneration. BPVT should be suspected in patients with clinical or echocardiographic evidence of BPV dysfunction, especially when presenting within five years after valve implantation. Elevated transvalvular gradients can be the first clue in diagnosing subclinical BPVT. Anticoagulation should be instituted in hemodynamically stable patients without contraindications before pursuing repeat valve replacement. Surgery should be reserved for non-responders to anticoagulation or patients in whom hemodynamic status precludes further delay. Although the optimal duration of anticoagulation is not known, long-term anticoagulation should be considered. In the event of cessation of oral anticoagulation, antiplatelet therapy and frequent echocardiographic surveillance can be considered.

\section{References:}

1. Puri R, Auffret V, Rodés-Cabau J: Bioprosthetic Valve Thrombosis. J Am Coll Cardiol. 2017; 24;69(17):2193-211.

2. Egbe AC, Pislaru SV, Pellikka PA, et al: Bioprosthetic Valve Thrombosis Versus Structural Failure: Clinical and Echocardiographic Predictors. J Am Coll Cardiol . 2015;66(21):2285-2294.

3. Nishimura RA, Otto CM, Bonow RO, et al: 2017 AHA/ACC Focused Update of the 2014 AHA/ACC Guideline for the Management of Patients With Valvular Heart Disease: A Report of the American College of Cardiology/American Heart Association Task Force on Clinical Practice Guidelines. J Am Coll Cardiol . 2017;70(2):252-289.

4. Baumgartner H, Falk V, Bax JJ, et al: 2017 ESC/EACTS Guidelines for the management of valvular heart disease. Eur Heart J . 2017;38(36):2739-2791.

5. Butnaru A, Shaheen J, Tzivoni D, et al: Diagnosis and treatment of early bioprosthetic malfunction in the mitral valve position due to thrombus formation. Am J Cardiol . 2013;112(9):1439-1444.

6. Oliver JM, Gallego P, Gonzalez A, et al: Bioprosthetic mitral valve thrombosis: Clinical profile, transesophageal echocardiographic features, and follow-up after anticoagulant therapy. Journal of the American Society of Echocardiography . 1996;9(5):691-699.

7. Egbe AC, Connolly HM, Pellikka PA, et al: Outcomes of Warfarin Therapy for Bioprosthetic Valve Thrombosis of Surgically Implanted Valves: A Prospective Study. J Am Coll Cardiol Intv . 2017;10(4):379387.

8. Petrescu I, Egbe AC, Ionescu F, et al: Long-Term Outcomes of Anticoagulation for Bioprosthetic Valve Thrombosis. J Am Coll Cardiol . 2020;75(8):857-866. 


\section{Figure legends:}

\section{Figure 1: Echocardiogram demonstrating mitral BPV echodensities (previous admission)}

Mitral BPV thickening and echodensities seen on (A) TTE (red arrow); (B) TEE (blue arrow); (C) 3D TEE and (D) TEE Transmitral gradients.

Figure 2 \& 3: Echocardiogram demonstrating mitral BPV thrombosis (current admission)

Mitral BPV thickening and echodensities seen on (2A) TTE (red arrow); (2B) TTE Transmitral gradients; (2C) TEE (blue arrow); (2D) TEE Transmitral gradients; (3A \& 3B) 3D TEE.

Figure 4: Echocardiogram demonstrating complete resolution of mitral BPV thrombosis after anticoagulation

Resolution of mitral BPV thickening and thrombosis after anticoagulation (A) TEE; (B) 3D TEE (C) TEE Transmitral gradients.

$\mathrm{BPV}=$ Bioprosthetic valve

$\mathrm{TTE}=$ Transthoracic echocardiogram

$\mathrm{TEE}=$ Transesophageal echocardiogram

$\mathrm{LV}=$ Left ventricle; $\mathrm{RV}=$ Right ventricle; $\mathrm{LA}=$ Left atrium; $\mathrm{RA}=$ Right atrium.

Video legends:

Video 1: TEE mid-esophageal four chamber view demonstrating mitral BPV thickening with mobile echodensities.

Video 2: 3D TEE mid-esophageal four chamber view showing mitral BPV thickening with mobile echodensity.

Video 3: TTE apical four chamber view showing mitral BPV thickening with mobile echodensities.

Video 4: TEE mid-esophageal four chamber view showing mitral BPV thickening with mobile echodensity.

Video 5: 3D TEE mid-esophageal four chamber view demonstrating mitral BPV thickening with mobile echodensity.

Video 6: 3D TEE mid-esophageal four chamber view demonstrating mitral BPV thickening with mobile echodensity.

Video 7: TEE mid-esophageal four chamber view demonstrating complete resolution of mitral BPV thrombosis after anticoagulation.

Video 8: 3D TEE mid-esophageal four chamber view demonstrating complete resolution of mitral BPV thrombosis after anticoagulation.

Consent: A written informed consent was obtained from the patient. 

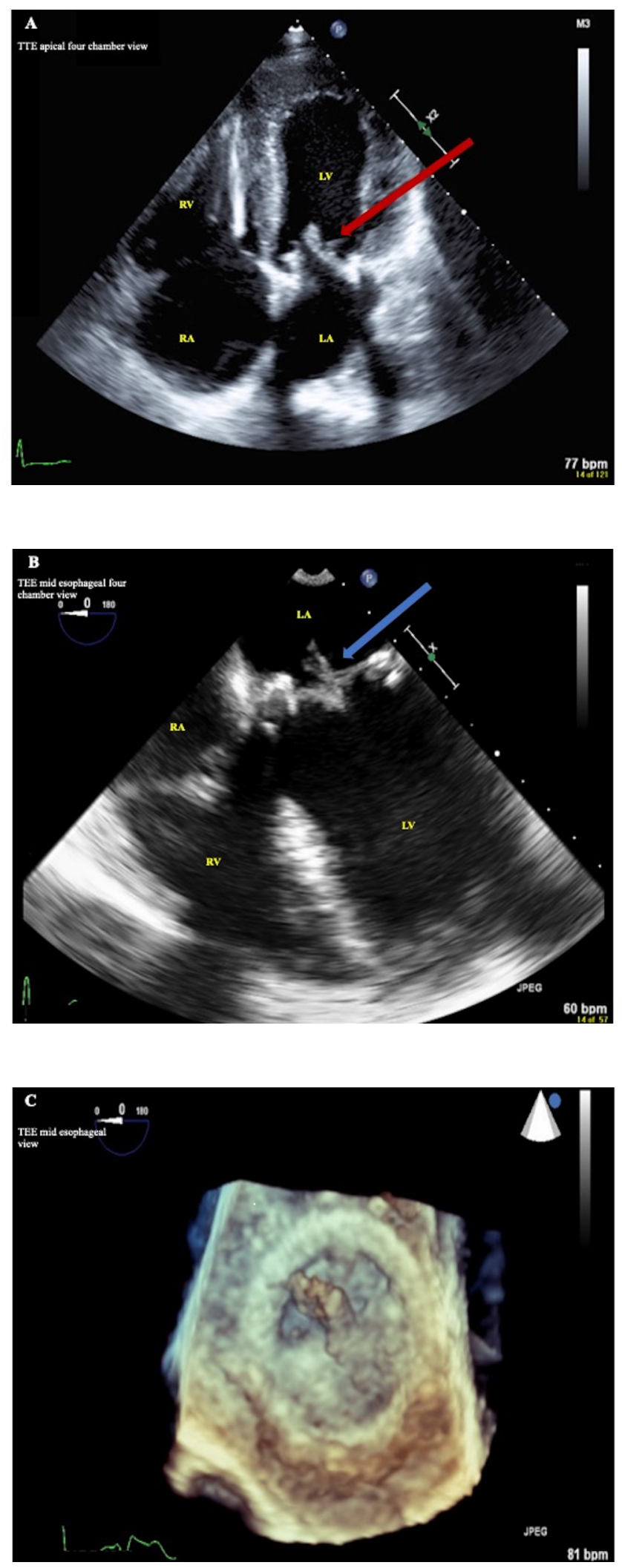

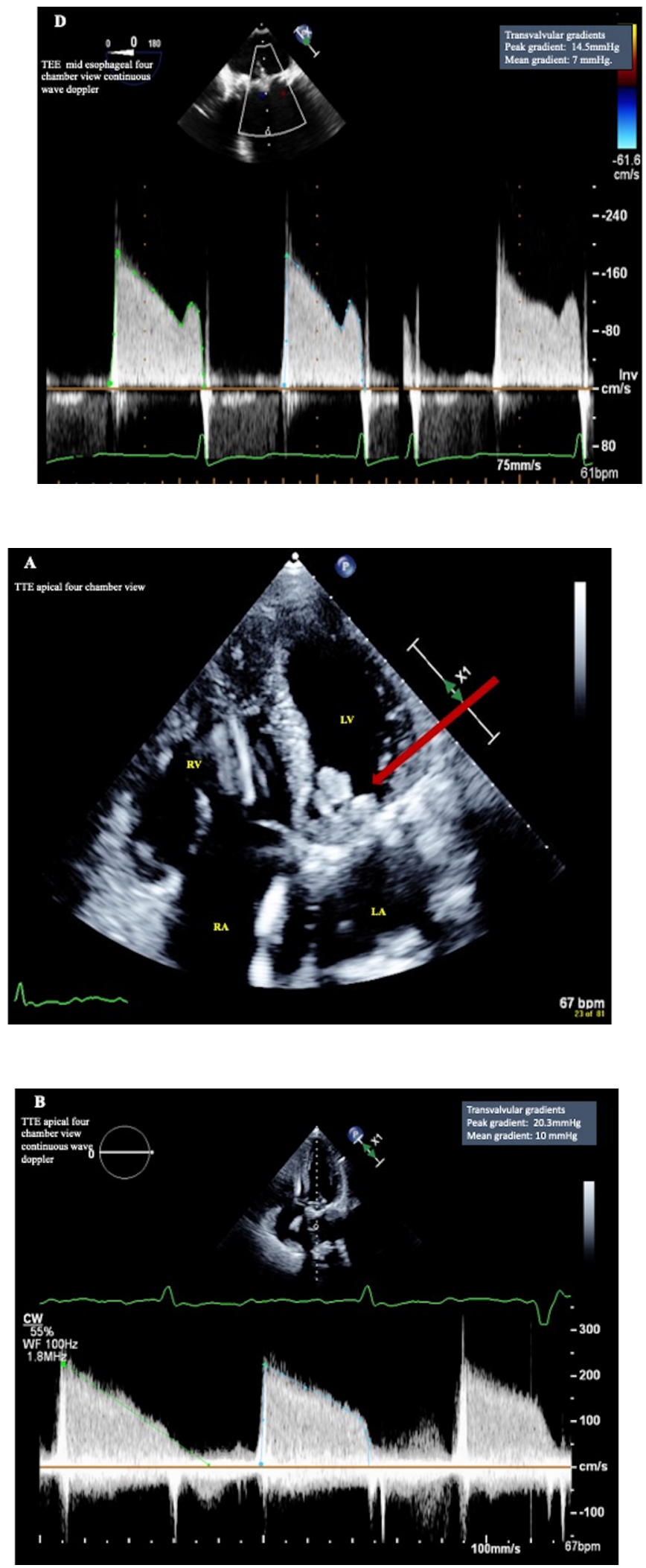

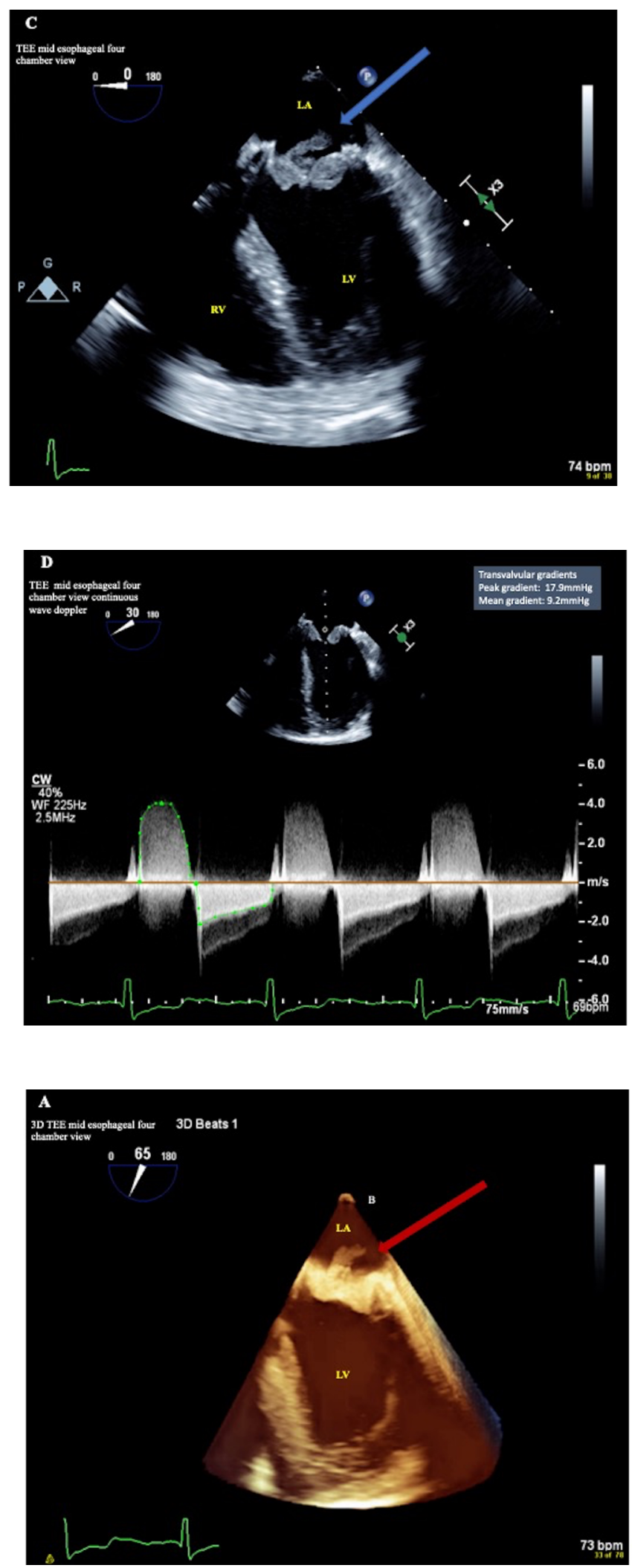

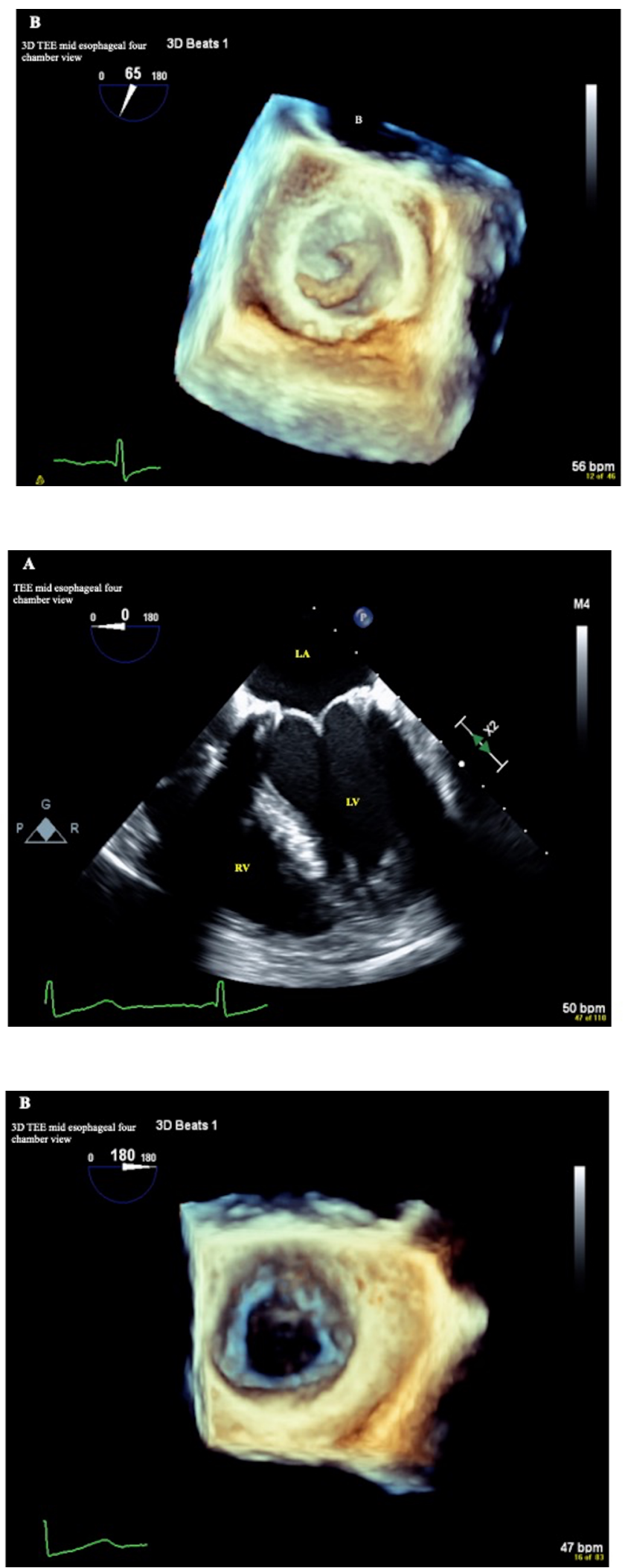


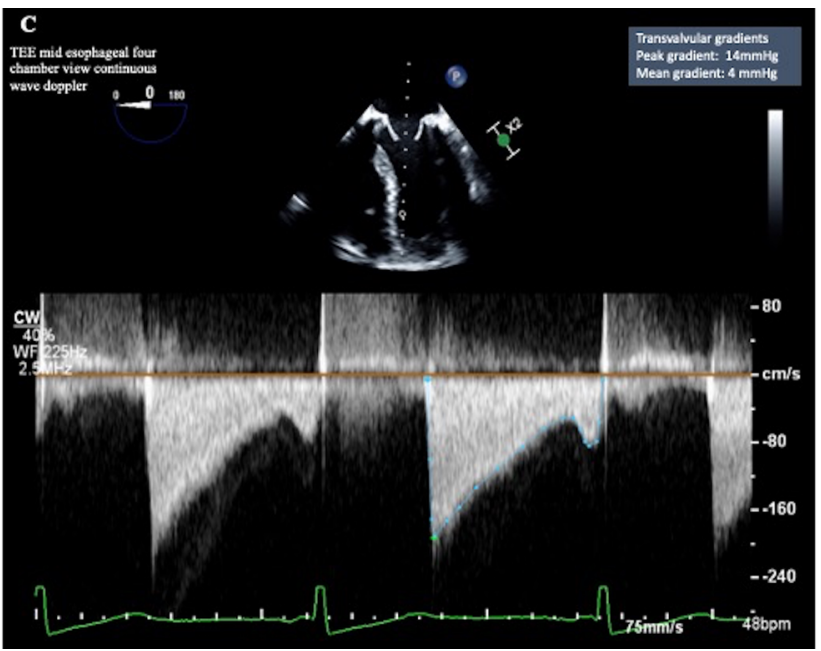

